\title{
Effects of Pre-Exhaustion of a Secondary Synergist on a Primary Mover in a Compound Exercise
}

\author{
Matthew J. Guarascio , Colin Penn , Cheryl Sparks* \\ Department of Physical Therapy, Bradley University, 1501 W. Bradley Avenue, Peoria, IL 61625, USA
}

Received: February 22, 2016; Accepted: April 28, 2016; Published: May 07, 2016

*Corresponding author: Cheryl Sparks, PhD, Bradley University, Department of Physical Therapy, 1501 W. Bradley Avenue, Peoria, IL 61625, USA, Office: (309)677-4533; Fax: (309)677-4053; E-mail: csparks@bradley.edu

\begin{abstract}
The purpose of this study was to investigate the effects of preexhausting a synergistic secondary mover prior to performing a compound exercise. Pre-exhaustion involves intentionally fatiguing a large muscle group in isolation prior to performing a compound exercise that utilizes the same muscle (e.g. performing glenohumeral joint horizontal adduction with a machine flying exercise immediately prior to barbell bench press in order to increase activation of pectoralis major. Surface electromyography was utilized to analyze the effects of pre-exhaustion of the triceps brachii on the pectoralis major while performing the barbell bench press. Data from the pectoralis major for 12 males were recorded during the barbell bench press; subjects performed this exercise with and without pre-exhaustion of the triceps brachii. A significant increase in electromyographic activity was noted in the pectoralis major $(\mathrm{z}=-2.353, \mathrm{p}=0.019)$ following pre-exhaustion of the triceps, indicating that pre-exhaustion of a synergistic secondary mover may result in higher neuromuscular activation of the primary mover in a compound exercise. Additional research with a larger sample size will provide more conclusive findings.
\end{abstract}

Keywords: Pre-Exhaustion; Neuromuscular Activation

\section{Introduction}

Exercise order is important to consider when designing a resistance-training program. Repetition performance [1, 2] strength $[3,4,5,6]$, force [7], power [7,8], and muscle volume $[3,4]$ have all been shown to be effected by the sequencing of exercises. Pre-exhaustion (PRE) is a method of exercise ordering that has been promoted in layperson bodybuilding literature. PRE involves intentionally fatiguing a large muscle group in isolation prior to performing a compound exercise that utilizes the same muscle (e.g. performing glenohumeral joint horizontal adduction with a machine chest flying exercise immediately prior to barbell bench press in order to increase activation of pectoralis major (PM)) [9]. While current research [10, 11, $12,13]$ on PRE has shown that this technique results in lower activation of the targeted muscle, it also demonstrated that PRE can result in increased neuromuscular activation of synergistic muscles $[11,12,13]$.
Subjects have performed the bench press with and without PRE of PM with a dumbbell chest flying exercise (2 dumbbells are simultaneously moved vertically from a position of shoulders horizontally abducted at $90^{\circ}$ to nearly $0^{\circ}$ )[12]. Electromyographic (EMG) data were recorded for the pectoralis major (PM), anterior deltoid (DA), and triceps brachii (TB). No significant changes in the tonic control of PM and DA were noted between the two treatment groups, however, TB did have a significantly $(\mathrm{p}<.05)$ higher motor unit activation when the chest was pre-exhausted with a dumbbell chest flying exercise.

Gentil et al. [11] compared upper-body muscle activation between PRE and the priority system, which involves performing complex exercises first in a strength-training program prior to performing single joint exercises. EMG data were recorded for TB, DA, and PM. The authors found TB activity to be significantly $(p<0.05)$ higher when the PRE method was used, however no difference was noted between the PRE and the priority system for DA and PM. Future investigation on the effects of fatiguing a synergistic muscle group prior to performing the primary exercise movement remains a research priority. Simao et al [14] echoed this in a review underscoring the importance of evaluating the effects associated with pre-exhausting muscle groups other than PM and the quadriceps.

There is a paucity of literature assessing the effects of preexhausting a synergistic secondary mover in isolation with a single-joint exercise prior to performing a compound lift. Therefore we investigated the effects of PRE of the triceps brachii prior to performing the barbell bench press, hypothesizing that this ordering of exercises will result in higher neuromuscular activation of the PM.

\section{Methods}

\section{Subjects}

Males between 20 and 35 years of age $(24, \pm 1.5$ years) were invited to participate. On average subjects weighed 88 $\pm 8 \mathrm{~kg}$, were $183 \pm 6.3 \mathrm{~cm}$ in height, and performed resistance training at least three times per week during the previous twelve 
months, with lifting experience that spanned $9 \pm 2$ years. Men were excluded from the study if they had a history of significant upper-body injury or any health conditions that presented with contraindications to participation in a strength-training program. All subjects were informed of the purpose, procedures, and risks associated with this study and were informed they could withdraw from participation at any time. Twelve men participated in the study.

\section{Experimental Design}

The 2-sequence, 2-period, 2-treatment balanced cross-over design performed by Gentile et al. [11]was replicated; however the washout period was extended from 20 to 30 minutes to 72 hours in order to ensure adequate recovery from the first testing session. Surface electromyography (EMG) was used to detect the neuromuscular activation of pectoralis major (PM) during two testing sessions: T1 (pre-exhaustion of triceps brachii prior to performing the bench press); T2 (only bench press). Group $\mathrm{AB}$ performed protocol $\mathrm{T} 1$, rested 72 hours, and then preformed protocol T2. Group BA performed protocol T2, rested 72 hours, and then preformed protocol T1 (Table 1).

\section{Electromyography}

EMG measurements were taken on the subjects' dominant side of the body. A $5 \mathrm{~cm}$ self-adhesive, circular, bipolar shielded, silver-silver chloride Triode electrode (Thought Technology Limited, Quebec, Canada) was utilized. Subjects were instructed to shave their chest prior to arrival. Recommendations from Florimond [15] were followed for anatomical placement of the electrodes. The site of electrode placement was identified and inspected by the same investigator. Tests were initiated once less than $5 \mu \mathrm{V}$ of resting EMG activity was observed [15].
Raw EMG signals were recorded using the Flex Comp Infinity encoder, and Myo Scan sensor (Thought Technology Limited, Quebec, Canada). Data were analyzed using Bio Graph Infiniti software (Thought Technology Limited, Quebec, Canada). The signal was recorded with a Common Mode Rejection $>130 \mathrm{~dB}$, and a fifth order Butterworth filter, with $30 \mathrm{~dB}$ alias rejection, was applied. Following this, the data were pre-amplified with a gain of 500 and band pass-filtered between 10 and $1000 \mathrm{~Hz}$, with a sample rate of 2048 samples/second.

\section{Determination of 10 Repetition Maximum Load}

Grip width for the bench press and triceps extension were normalized by subjects' bi-acromial width. Subjects were instructed on proper lifting technique, and an automated metronome was played to assist subjects in following a 2-0-2 (eccentric, isometric, concentric) tempo. Subjects performed the barbell bench press at a weight that they estimated they could lift approximately 10 times without compromising technique or rate. If subjects performed more or less than 10 repetitions, the weight was adjusted by 5 -pound increments. 5 minutes of rest were taken between sets. A minimum of one and a maximum of three attempts were performed. A 20-minute rest period followed, and then the same protocol was repeated for the triceps extension exercise. Subjects were asked to not make any changes to their normal levels of physical activity, with the exception of not exercising the chest, triceps, or anterior shoulders for 72 hours prior to their testing sessions.

\section{Experimental Protocol}

Subjects warmed up on the bench press with 10-15 repetitions at $50 \%$ of their estimated 10 -repetition maximum load. Three maximum voluntary isometric contractions (MVIC) of the PM were performed against a fixed resistance with shoulders

\begin{tabular}{|c|l|c|c|c|}
\hline Group & $\begin{array}{l}\text { 10 Rep } \\
\text { max Test } \\
\text { Day } \\
\text { (Triceps } \\
\text { Extension }+ \\
\text { Bench } \\
\text { Press) }\end{array}$ & $\begin{array}{c}\text { Maximal } \\
\text { Voluntary } \\
\text { Isometric } \\
\text { Contraction } \\
\text { (each test } \\
\text { day) }\end{array}$ & $\begin{array}{c}\text { Bench } \\
\text { Press } \\
\text { without } \\
\text { PRE }\end{array}$ & $\begin{array}{c}\text { Bench } \\
\text { Press with } \\
\text { PRE }\end{array}$ \\
\hline AB & $1^{\text {st }}$ day & Yes & $2^{\text {nd }}$ day & $3^{\text {rd }}$ day \\
\hline BA & $1^{\text {st }}$ day & Yes & $3^{\text {rd }}$ day & $2^{\text {nd }}$ day \\
\hline
\end{tabular}

Table 1: Protocol for the two different groups 
flexed to $90^{\circ}$, elbows extended to $0^{\circ}$, and forearms pronation. A one second ramp up period was followed by 4 seconds of maximal contraction. Subjects rested for 20 seconds between repetitions, and for five minutes before beginning testing. For protocol T1, subjects performed the bench press exercise at their previously determined 10 repetition maximum load. For protocol T2, subjects performed one set of a triceps extension exercise to fatigue at the previously determined 10 repetition maximum load, and immediately moved to the bench press to perform repetitions to fatigue at the previously determined 10 repetition maximum load. In both protocols, testing ceased if the participants' form was compromised or if they were unable to maintain proper tempo. EMG data were collected for the PM during the barbell bench press for both protocols. Subjects were asked to not make any changes to their normal levels of physical activity, with the exception of not exercising the chest, triceps, or anterior shoulder for 72 hours prior to performing the alternative protocol.

\section{Data Analysis}

Raw EMG data were analyzed using Biograph Infiniti software (version 5.1.2).The average signal amplitude was calculated using the root mean square method. A blinded investigator identified peak MVIC values according to protocol established by Thought Technology[15].The first and last repetitions were visually identified and removed from the dataset to eliminate artifact caused by the racking and unracking of the bar. The amplitude was normalized to the mean of the peak EMG values obtained during the MVIC tests for each subject.

\section{Statistical Analysis}

Normalized RMS values for exercise protocol T1 and T2 were compared using the Wilcoxon signed rank test. Total repetitions, mean time per counted repetition, and mean MVIC were compared between groups $\mathrm{AB}$ and $\mathrm{BA}$ using a paired samples t-test. All results are presented in values of mean \pm SD; an alpha level of 0.05 was used for all comparisons.

\section{Results}

PM activation was significantly higher in the PRE group than the no-PRE group during the bench press exercise $(\mathrm{Z}=$ $-2.353, p=.019$ ) (Figure 1) Between group comparisons of total repetitions $(\mathrm{t}(11)=1.036, \mathrm{p}=.322)$, mean time per counted repetition $(\mathrm{t}(11)=-1.470, \mathrm{p}=.170)$, and mean MVIC $(\mathrm{t}(11)=.499$, $\mathrm{p}=.628)$ were not significant $(\mathrm{p}>0.05)$.

\section{Discussion}

Results indicated that pre-exhaustion of TB with a triceps extension exercise could result in higher neuromuscular activation of PM during a bench press exercise than when no preexhaustion is performed. The observed altered recruitment of a primary mover after PRE of a synergistic secondary mover is similar to the outcome of other studies $[11,12,13]$, which showed that PRE of a primary mover could result in altered recruitment patterns of synergistic muscles. Gentil et al. [11] and Brennecke et al. [12] pre-exhausted pectoralis major before the performance of the bench press, and both authors observed a significant $(\mathrm{p}<$ .05) change in the neuromuscular activation of TB.Akima et al. [13]demonstrated increased activation of vastusmedialis and rectus femoris after PRE of VL through electromyo stimulation.

A review of current research on exercise ordering in resistance training performed by Simão et al. [14] concluded that if an exercise is important it should be placed at the beginning of the exercise sequence. Our findings suggest that future research investigating the chronic effects of PRE of a synergistic secondary mover prior to performance of a compound exercise may reveal an alternative, and perhaps more appropriate, form of exercise sequencing than the current recommendations. Miranda et al. [16] investigated all possible ordering sequences for bench press (BP), triceps extension (TE), and shoulder press (SP) and concluded that the only significant effect on repetition performance occurred during the $\mathrm{SP}, \mathrm{TE}, \mathrm{BP}$ sequence when compared to the TE, BP, SP and the TE, SP, BP sequences. The $\mathrm{SP}, \mathrm{TE}, \mathrm{BP}$ sequence resulted in significantly less repetitions

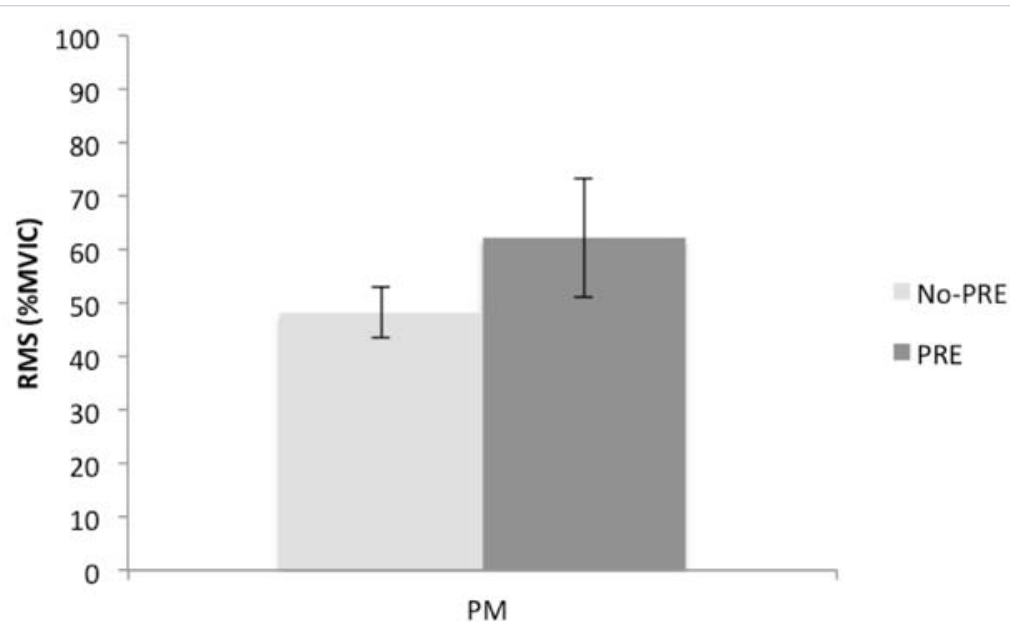

Figure 1: Comparison between normalized RMS values for pectoralis major during bench press exercise for protocol T1 (pre-exhaustion of triceps brachii prior to performing bench press) and T2 (no pre-exhaustion prior to performing bench press). 
performed. Therefore, placing TE prior to BP might not affect repetition performance during a three-exercise sequence. In addition to conducting a long-term study on the chronic effects of PRE of a secondary synergist, future research should also investigate the acute affects of this exercise sequence on additional local musculature such as the anterior deltoid, and triceps brachii.

Previous research [11, 12] assessing the effects of preexhausting pectoralis major with a single-joint exercise prior to performing the barbell bench press demonstrated that the PRE method is not effective in accomplishing the goal promoted in layperson bodybuilding literature. We incorporated subjects of similar age and lifting experience to these studies $[11,12]$ and found that the goal of PRE can possibly be accomplished by preexhausting the TB prior to the barbell bench press. Additional inquiry with a larger sample will provide more conclusive findings.

\section{Acknowledgements}

The authors would like to thank Bradley University Department of Physical Therapy and Health Science, Alex Barry, and Brandon Coates for assistance with data collection, and Joseph Kelly, PhDC, PT for his guidance related to this project.

\section{References}

1. Miranda $\mathrm{H}^{1}$, Simão $\mathrm{R}$, dos Santos Vigário $\mathrm{P}$, de Salles $\mathrm{BF}$, Pacheco MT, Willardson JM. Exercise order interacts with rest interval during upper-body resistance exercise. J Strength Cond Res. 2010;24(6):15731577. doi:10.1519/JSC.0b013e3181d8ea61.

2. Simão $\mathrm{R}^{1}$, Farinatti Pde T, Polito MD , Maior AS, Fleck SJ. Influence of exercise order on the number of repetitions performed and perceived exertion during resistance exercises. J Strength Cond Res. 2005;19(1):152-156.

3. Simão $\mathrm{R}^{1}$, Spineti J, de Salles BF, Oliveira LF, Matta T, Miranda F,et al Influence of exercise order on maximum strength and muscle thickness in untrained men. J Sports Sci Med. 2010;9(1):1-7. eCollection 2010.

4. Spineti $\mathrm{J}^{1}$, de Salles BF, Rhea MR, Lavigne D, Matta T, Miranda F,et al.Influence of exercise order on maximum strength and muscle volume in nonlinear periodized resistance training. I Strength Cond Res. 2010;24(11):2962-9. doi: 10.1519/JSC.0b013e3181e2e19b
5. Assumpção $\mathrm{CO}^{1}$, Tibana RA, Viana LC, Willardson JM, Prestes J. Influence of exercise order on upper body maximum and submaximal strength gains in trained men. ClinPhysiol Funct Imaging. 2013;33(5):359-363. doi: 10.1111/cpf.12036. Epub 2013 Mar 6.

6. Dias $I^{1}$, de Salles BF, Novaes J, Costa PB, Simão R. Influence of exercise order on maximum strength in untrained young men. J Sci Med Sport. 2010;13(1):65-69. doi: 10.1016/j.jsams.2008.09.003. Epub 2009 Feb 25.

7. Baker $\mathrm{D}^{1}$, Newton RU. Acute effect on power output of alternating an agonist and antagonist muscle exercise during complex training. J Strength Cond Res. 2005;19(1):202-205.

8. Spreuwenberg LP ${ }^{1}$, Kraemer WJ, Spiering BA, Volek JS, Hatfield DL, Silvestre $\mathrm{R}$, et al.Influence of exercise order in a resistance-training exercise session. J Strength Cond Res. 2006 ;20(1):141-144.

9. Baechle TR, Earle RW. Essentials of Strength Training and Conditioning. 3rd ed. Champaign: Human Kinetics; c2008. 656 p.

10.Augustsson $\mathrm{J}^{1}$, Thomeé R, Hörnstedt $\mathrm{P}$, Lindblom J, Karlsson J, Grimby G. Effect of Pre-Exhaustion Exercise on Lower-Extremity Muscle Activation During a Leg Press Exercise. J Strength Cond Res. 2003;17(2):411-416.

11. Gentil $\mathrm{P}^{1}$, Oliveira E, de Araújo Rocha Júnior V, do Carmo J, Bottaro M. Effects of exercise order on upper-body muscle activation and exercise performance. J Strength Cond Res. 2007;21(4):1082-1086. ?

12. Brennecke $A^{1}$, Guimarães TM, Leone R, Cadarci M, Mochizuki L, Simão $\mathrm{R}$, et al.Neuromuscular activity during bench press exercise performed with and without the preexhaustion method. J Strength Cond Res. 2009;23(7):1933-1940.

13. Akima $\mathrm{H}^{1}$, Foley JM, Prior BM, Dudley GA, Meyer RA. Vastuslateralis fatigue alters recruitment of musculus quadriceps femoris in humans. J ApplPhysiol 2002;92(2):679-684.

14.Simão $\mathrm{R}^{1}$, de Salles BF, Figueiredo T, Dias I, Willardson JM. Exercise order in resistance training. Sports Med. 2012 Mar 1;42(3):251-65.

15. Florimond V. (2009 \& 2010). Basics of Surface Electromyography Applied toPhysical

16. Rehabilitation and Biomechanics [Internet]. Montreal: Thought Technology Limited; 2009 [modified 2010; cited 2015 Mar 11]. 50 p. Available from: http://thoughttechnology.com/index.php/ documentation. 